\title{
SIMULACIÓN CON ECOCARDIOGRAFÍA TRANSESOFÁGICA COMO MÉTODO DE ENSEÑANZA
}

\author{
María Carolina Cabrera Schulmeyer ${ }^{1}$, Rocio Armesto ${ }^{2}$, Daniel Marcelo Campos ${ }^{3}$, Mario Valotta ${ }^{3}$, Ariel Bonardi ${ }^{3}$, \\ Gustavo Abuin ${ }^{3}$ \\ Universidad de Valparaíso, sede hospital Fach. \\ Universidad de Valparaíso, residente. \\ AAARBA, Argentina.
}

Introducción: La ecocardiográfica (ETE) transesofágica perioperatoria es una herramienta de monitorización y diagnostico que ha demostrado utilidad tanto en cirugía cardiaca como no cardiaca. Esto ha generado alto interés de los anestesiólogos por entrenarse y formarse. Como un aporte para lograr objetivo se diseñó un taller práctico utilizando simulador de ETE.

Objetivo General: Evaluar el aprendizaje de la técnica con una sonda transesofágica multiplanar a través de la enseñanza de cortes anatómicos estandarizados con el simulador y clases teóricas relacionadas al tema.

Material y Métodos: Se obtuvo el consentimiento de 85 alumnos sin conocimiento previo en ecocardiografía, para estudiar su aprendizaje antes y después del curso-taller. Antes de asistir a las clases teóricas y ser expuestos a la simulación se les realizó un test de 15 preguntas de múltiple elección, basadas en videos de los diferentes cortes anatómicos normales. No se evaluó estructuras patológicas y tampoco cálculos hemodinámicos. Este examen fue realizado con un orden diferente al final del entrenamiento siguiendo la misma modalidad con los 15 videos. Cada alumno completó una encuesta de satisfacción. Al inicio se realizó una demostración con las características de la sonda transesofágica para adultos, se demostró su forma de introducirla y el funcionamiento del sistema multiplanar. A continuación y siguiendo un esquema de cortes que cada alumno tenía impreso, se mostraron en el simulador de ecocardiografía, los cortes en el mismo orden e identificando cada estructura anatómica normal.

Resultados: Del total de 85 alumnos, 80 participaron en el estudio, ya que 5 no alcanzaron a rendir el test inicial por razones de su horario laboral. La edad promedio fue de $32 \pm 9$ años, 49 hombres, con un promedio de años ejerciendo como anestesiólogo de $6 \pm 0,8$ años. En la evaluación inicial sólo el 23\% alcanzó más de 12 preguntas correctas, mientras que en la evaluación final un $85 \%$ alcanzó más de 12 preguntas correctas $(\mathrm{p}<0,01)$. En cuanto a la satisfacción de los alumnos un $85 \%$ estuvo muy de acuerdo con la forma de aprender ETE, un 12\% estuvo de acuerdo y sólo el 3\% no estuvo de acuerdo ni en desacuerdo.

Conclusiones: La ETE se realiza a pacientes complejos en condiciones inestables, así las oportunidades para el aprendizaje sin stress son pocas. Un taller con simulador de ETE, mejorara estas condiciones y así el conocimiento del alumno. Esto se evidenció en el aumento de respuestas correctas en la evaluación antes y después de realizar la simulación. El aprendizaje de la ecografía transesofágica requiere de la comprensión cognitiva de la anatomía cardiaca normal y patológica la imaginación de la posición tridimensional de la sonda el plano de exploración del corazón y los grandes vasos y la habilidad sicomotriz para la inserción y manipulación del transductor y esto se facilita de manera importante utilizando el simulador con una alta satisfacción de los alumnos. 